\title{
ROLF HERZOG
}

\section{Ethnographische Sammlungen, Lehr- und Forschungsstätten in Kairo}


VERÖFFENTLICHUNGEN

DES MUSEUMS FÜR VÖLKERKUNDE ZU LEIPZIG HEFT 11

\title{
BEITRÄGE ZUR VÖLKERFORSCHUNG
}

\author{
HANS DAMM ZUM 65. GEBURTSTAG
}

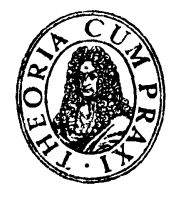

AKA D E M I E - VER LAG - B E R L I N 


\title{
ETHNOGRAPHISCHE SAMMLUNGEN, LEHR- UND FORSCHUNGS- STÄTTEN IN KAIRO
}

\author{
Von Rolf Herzog, Kairo
}

In der Hauptstadt der Vereinigten Arabischen Republik gibt es kein Völkerkundemuseum, sondern die hier zusammengetragenen Ethnographika sind über verschiedene Sammlungen verteilt oder lagern in Magazinen. Es mag deshalb Interesse finden, die wesentlichsten Bestände wenigstens aufzuzählen und kurz zu charakterisieren. Mancher Fachkollege, dem nur ein flüchtiger Aufenthalt in Ägypten vergönnt ist, mag vielleicht diese Übersicht bei seiner Programmgestaltung nutzbringend verwerten können. Die Beifügung der Straßenbezeichnung bei weniger bekannten Instituten soll ebenfalls der Praxis dienen.

Als bedeutendste Sammlung ist die der Geographischen Gesellschaft (Sharia Qasr el-'Aini) zu nennen. 1875 von Georg Schweinfurth gegründet, hat diese Institution von vielen Reisenden aus dem Niltal in seiner gesamten Ausdehnung, d. h. einschließlich des Sudans und Ugandas, Belegstücke der materiellen Kultur der Eingeborenen aufgenommen. Wegen Raummangels und dringlich gewordener Erweiterung der Stellfläche für die von Jahr zu Jahr anschwellende Bibliothek wurde die ethnographische Sammlung schließlich aus ihren Räumen entfernt und im Erdgeschoß sehr eng, fast magazinartig untergebracht. Nur nach vorheriger Vereinbarung mit dem Bibliothekar ist die Besichtigung möglich. Die alte Aufstellungsordnung war die Grundlage für E. S. Thoмas' „Catalogue of the ethnographical Museum of the Royal Geographical Society of Egypt", erschienen im Bulletin de la Société de Géographie d'Egypte, t. 12 u. 13, Kairo 1924. Der Verfasser war kein Völkerkundler, sondern vordem mit der Katalogisierung von Altertümern beschäftigt gewesen. Das mag einen Teil der nicht geglückten oder zu allgemeinen Beschreibungen in seiner Übersicht erklären. Immerhin ist jedoch von Thomas damit die einzige größere Kairiner ethnographische Sammlung, welche heute rund 1500 Nummern umfaßt, publiziert worden.

Wesentlich jünger sind die Stücke der heimatkundlichen Abteilung im breit angelegten Landwirtschaftsmuseum im Stadtteil Doqqi. Die Ausstellungstechnik ist bewußt darauf ausgerichtet, ein nicht vorgebildetes Publikum anzusprechen. So sind lebensechte Figuren in natürlicher Größe und Originaltracht aufgestellt, um einheimisches Handwerk, Kaffeehaustreiben, Hochzeit und vieles andere aus 
dem täglichen Leben naturgetreu darzustellen. Wer selbst mit ansieht, wie sich die Daseinsform aller Schichten der Ägypter, besonders der Städter, rasch modernisiert, wird diesen Museumsteil, dessen Sinn und Aufgabe 'ABD ER-RAZIK SIDKY $^{1}$ umrissen hat, willkommen heißen. Auch andere Abteilungen dieses $\mathrm{Mu}$ seums enthalten für den Völkerkundler Interessantes, z. B. Sattelzeuge, Flechtarbeiten, Fischerboote, Netze, Brotformen und Backöfen, eine stattliche Kollektion von Pflügen, Pflugmodellen, landwirtschaftlichem Arbeitsgerät und Bewässerungsvorrichtungen.

Im Geographischen Institut der Universität von Giza (Midan er-Ramah) ist eine kleine Sammlung, die der vor wenigen Jahren verstorbene Professor LUDwIG KEIMER im Oberägypten erwarb, in Vitrinen ausgestellt. Die meisten Stücke stammen von den Bischarin.

Das Wüsteninstitut (Desert Institute) im Vorort Mataria besitzt ebenfalls eine kleine ethnographische Sammlung, die vorwiegend in den Oasen zusammengetragen wurde. Als diese Forschungsstelle noch in ihrem eigenen Gebäude in Heliopolis residierte, erschien jeweils ein Teil der Kollektion in Wechselausstellungen. Leider ist der frühere, sehr ansprechende Bau inzwischen zweckentfremdet worden. In dem völlig ungeeigneten ehemaligen prinzlichen Palast in Mataria verschwanden die Ethnographika in Kisten, und es bedarf langwieriger $\mathrm{Ab}$ machungen, sie wenigstens vorübergehend ans Licht $\mathrm{zu}$ bringen. Ebenso unerreichbar verschlossen bleibt z. Z. eine sudanische Kollektion im Afrika-Institut.

Eine vorerst noch bescheidene, aber durch ständigen Zukauf wachsende Zahl von Belegstücken ländlicher Trachten und Gebrauchsgegenständen findet man im Center of Folklore (Sharia 'Abd el-Khalik Sarwat Pasha 27).

Das beachtliche Museum für Islamische Kunst am Bab el-Khalk enthält neben vielen Stücken, die eng mit der Religionsausübung verknüpft sind, auch manches aus dem täglichen Leben der Moslime. Ebenso bewahrt das Koptische Museum in Alt-Kairo eine kleine Zahl von Gebrauchsgegenständen, die z. T. Jahrhunderte alt sein dürften, in einem Nebenflügel auf, der auf Verlangen bereitwillig geöffnet wird. Diese beiden großen Museen, deren Besuch ein Völkerkundler nicht versäumen sollte, unterscheiden sich ausstellungstechnisch vorteilhaft von dem noch umfangreicheren Altertümermuseum am Midan et-Tahrir. Letzteres ist wegen seiner kaum überbietbaren Überfüllung, der Anhäufung gleichartiger Dubletten, der unzureichenden Beschriftung und fürchterlichen Enge eher ein zur Besichtigung freigegebenes Magazin. Wer mit Museumsfragen vertraut ist, wird rasch feststellen, wie hier weltberühmte Schätze, z. B. der Grabfund des Tut-anchAmon, durch unzulängliche Ausstellungsordnung verlieren und der Besuch keineswegs erquicklich wird. Völkerkundlich ist die nubische Abteilung, die viele Grabbeigaben aus relativ später Zeit zeigt, von Interesse.

1 'Abd Er-Razik Sidky: Das neue Heimatmuseum in Kairo. Cahiers d'Histoire Egyptienne. Sér. IX, fasc. 1/2, Kairo 1957. 
Zwei militärische Museen schließen Sammlungen von Waffen und Kriegsausrüstungen einiger Stämme ein. Das größere der beiden auf der Zitadelle enthält in einem Nebenflügel einige Räume mit Stücken aus dem Sudan. Im kleineren, im rückwärtigen Teil des Abdin-Palastes, ist weniger dergleichen zu finden.

Die Wohnverhältnisse Kairiner Bürger von etwa der Art wie sie LANE in seinen „Manners and customs of the modern Egyptians" 1836 schilderte, bietet, wenn auch in sich nicht einheitlich, das Guyer-Anderson-Museum, arabisch Beit elKiridliya genannt. Der Gründer lebte als Arzt und Liebhaber-Sammler in einem alten Haus, das in die Ostecke der Umfassungsmauer der berühmten Ibn-TulunMoschee eingebaut ist. Seit wenigen Jahren ist es zum Besuch freigegeben.

Schwerer zu finden ist ein mit seiner alten Inneneinrichtung erhaltenes Gebäude, das lange als Dienstwohnung für Lehrkräfte der über tausendjährigen al-Azhar-Universität diente. Die volkstümliche Bezeichnung ist Menzel essuhaimi. Man läßt sich am besten von dem Wächter der Ibn-Qala'un-Moschee, nicht weit vom Basar Khan el-Khalili, einen Jungen als Begleiter mitgeben, um im Gewirr der Gassen dieses enggebauten Viertels den Weg nicht zu verlieren.

Neben den bisher aufgezählten Museen und Sammlungen gibt es natürlich noch andere, von denen einige auch Völkerkundler ansprechen mögen. Oft haben sie einzelne Objekte von Bedeutung. Das Gezira-Museum auf der Nilinsel Zamalek enthält, um ein Beispiel anzuführen, viele erstklassige Proben des Kunsthandwerks nicht nur Ägyptens, sondern der arabischen Welt im weiteren Sinne. Dazwischen geraten ist eine prächtige alte Kaffeeröstkelle von Beduinen.

$\mathrm{Zu}$ den jetzt bestehenden Museen dürften noch weitere hinzutreten. Einer Zeitungsmeldung ${ }^{2}$ zufolge ist die Errichtung eines Schiffahrtsmuseums vom Kulturminister verfügt worden. In ihm soll auch die alte Seefahrt der Araber, z. B. auf dem Roten Meere, ihren Platz finden. - Einige einflußreiche Persönlichkeiten des Kairiner Geisteslebens versuchen, ein zentrales ethnographischheimatkundliches Museum durchzusetzen. Welcher Erfolg ihrer lobenswerten Bemühung beschieden sein wird, muß die Zukunft lehren.

Ägypten zählt fünf Universitäten, wenn man die religiöse al-Azhar wegläßt, nämlich drei in Kairo (Giza, 'Ain Shams und die amerikanische), dazu je eine in Alexandrien und Asiut. An keiner besteht z. Z. ein Lehrstuhl für Völkerkunde. Dr. Aнмеd Aвu ZeID hält in Alexandria im Rahmen der Soziologie völkerkundliche Vorlesungen; seine Position entspricht etwa der eines außerplanmäßigen Professors. An der amerikanischen Universität arbeiten einige Wissenschaftler, die meist aus dem Nahen Osten stammen, im Social Research Center an Vorhaben, die z. T. völkerkundliche Fragen einbeziehen. Der Schwerpunkt liegt aber auf der Soziologie.

2 Egyptian Gazette vom 24. Januar 1960. 
Der Universität Giza angeschlossen ist das Institute of African Studies (Sharia el-Mesaha 5, Doqqi), das in den ersten Jahren seines Bestehens (gegründet 1949) als Institute of Sudanese Studies bezeichnet wurde. An ihm werden sogenannte post-graduates, also Studenten, die den untersten akademischen Grad - Bachelor of Arts zum Beispiel - schon erlangt haben, weitergebildet. Neben Geographie und Geschichte schließt der Lehrplan eine völkerkundliche Vorlesung ein, die dẹr in Wien ausgebildete Dr. Mohammed Riad hält. An diesem Institut wirkt auch Prof. Mohammed Awad, ein Geograph, der mehrfach zu anthropogeographischen Themen publiziert hat.

Das dem Kultusministerium unterstehende Center of Folklore bemüht sich, Sitte und Brauchtum aufzunehmen, in der richtigen Erkenntnis, daß vieles davon im Verschwinden begriffen ist und vielleicht schon in der nächsten Generation kaum noch deutlich faßbar sein mag. So nahm man z. B. Hochzeitsbräuche verschiedener Landschaften, 1959 auch das Brauchtum des Fastenmonats Ramadan auf. Hinzu kommen Tonbandbelege von Volksmusik. Die Angestellten des Centers, ausnahmslos Ägypter, haben gelegentlich gegen Verständnislosigkeit der provinziellen Behörden anzukämpfen. Eine im Februar 1960 bereits eingeleitete Erfassung derjenigen Bischarin, die noch nicht in der Vorstadt Aswans seßhaft sind, wurde vom örtlichen Kommandanten der Grenzwache, der nicht nur jede Hilfe verweigerte, sondern alle nur erdenklichen Hemmnisse in den Weg stellte, vereitelt.

Das Wüsteninstitut in Mataria hat vor einigen Jahren die Anregung zu verwirklichen versucht, das Studium der Beduinenstämme in sein Arbeitsprogramm einzubeziehen. Doch ist dem guten Willen nur die halbe Tat gefolgt, teils wohl, weil qualifizierte einheimische Kräfte dafür nicht zu gewinnen waren, teils weil häufiger Wechsel in den leitenden Stellungen vielfältig abgeänderte Ansichten nach sich zog. So beherrschen bis heute die Naturwissenschaften, vorwiegend Botanik, Geologie und Hydrologie, das Institut. Manches davon, wie Untersuchungen über Weideflächen, Grasarten wie deren Dürrefestigkeit oder Grundwasserveränderungen, sind für Ethnologen, die sich dem Nomadentum speziell zuwenden, nicht ohne Bedeutung. Es ist z. Z. nicht abzuschätzen, ob das Institut in naher Zukunft den letzten echten Wüstenbewohnern, so muß man es im Hinblick auf die rasche Abnahme des Nomadenbestandes formulieren, noch rechtzeitig seine Aufmerksamkeit schenken wird. Es ist zu befürchten, daß - wie auch in anderen Staaten mit einem beträchtlichen Prozentsatz arider Fläche - die naturwissenschaftliche Erforschung der Wüste ebenso schnell wie deren Entvölkerung fortschreiten wird.

Einige der eben beschriebenen Institutionen geben Publikationen heraus. Im Institute of African Studies hat der rührige Bibliothekar, vorher jahrzehntelang im Sudan tätig, mehrfach zur Feder gegriffen; Shater Busaiti (auch Chater BosayLaY transkribiert) wandte sich vorwiegend historischen Themen zu. In den 
letzten Jahren hat er nur in Arabisch veröffentlicht. - Das Center of Folklore hat das erste Heft seiner Zeitschrift „Folklore“ teils mit Beiträgen in europäischen Sprachen, teils mit solchen in Arabisch bestritten. - Das schon zu stattlicher Bandzahl gediehene Bulletin de la Société de Géographie d'Egypte brachte in früheren Jahren viel häufiger völkerkundliche Beiträge als gegenwärtig. Man kann bei nicht weniger traditionsreichen Zeitschriften, auch bei Petermanns Mitteilungen, beobachten, daß die älteren Jahrgänge einen beträchtlich weiteren Rahmen umspannten als die jetzigen. Das mag auf der Linie der scheinbar unvermeidlichen Spezialisierung aller Wissenschaften liegen. - Nach dem vorher Gesagten ist es klar, daß das Bulletin de l'Institut du Désert wie auch seine unter dem Reihentitel Publications laufenden Abhandlungen überwiegend naturwissenschaftlich orientiert sind. - Verstreut finden sich noch einige völkerkundlich inhaltsreiche Aufsätze im Bulletin of the Faculty of Arts, Cairo University, in den Cahiers d'Histoire égyptienne oder dem Bulletin de l'Institut d'Egypte.

Im UNESCO Middle East Office in Kairo (Sharia es-Salamlik 8, Garden City) wird wertvolle bibliographische Titelerfassung geleistet, einmal in den unregelmäßig erscheinenden Bibliographical Lists of Social Sciences Publications, zum anderen in zusammenfassenden Verzeichnissen, wie der kürzlich ausgegebenen Retrospective Bibliography of Social Science Works published in the Middle East, U.A.R., Iraq, Jordan, Lebanon, 1945-1955.

Weder die Nationalbibliothek am Bab el-Khalk, noch die Universitätsbibliothek enthalten größere Buchbestände, die völkerkundlichen Studien dienen könnten. Dagegen ist die Bibliothek der Geographischen Gesellschaft eine Fundgrube für Werke über das Niltal. Das dicht daneben untergebrachte Institut d'Egypte verwahrt manches seltene ältere Buch über die Völker Nordostafrikas. Gut ausgestattet ist auch die Bücherei des Institut Français d'Archéologie Orientale (Sharia esh-Sheikh Ali Yussuf, Munira). Schließlich sei noch vermerkt, daß eine kostbare Sammlung alter Reisebeschreibungen, die der bibliophile Spürsinn Ludwig KeImers in Jahrzehnten zusammentrug, im Deutschen Ärchäologischen Institut (Sharia Gezira el-Wusta 22, Zamalek) jedem Gelehrten zugängig ist. Die völkerkundliche Sektion dieses Instituts, an der der Verfasser dieses Beitrages wirkt, ergänzt den Bestand an Itinerarien durch Anschaffung neuester Veröffentlichungen über die Menschen am Nil, im islamisierten Afrika und im Nahen Osten. 